\title{
Singular Levi-flat hypersurfaces and codimension one foliations
}

\author{
MARCO BRUNELlA
}

\begin{abstract}
We study Levi-flat real analytic hypersurfaces with singularities. We prove that the Levi foliation on the regular part of the hypersurface can be holomorphically extended, in a suitable sense, to neighbourhoods of singular points.
\end{abstract}

Mathematics Subject Classification (2000): 32V25 (primary); 32S65, 32C05 (secondary).

\section{Introduction}

Let $X$ be a complex manifold of dimension $n$. A smooth real hypersurface $M \subset X$ is said to be Levi-flat if the codimension one distribution

$$
T^{\mathbb{C}} M=T M \cap J(T M) \subset T M
$$

is integrable, in Frobenius' sense. It follows that $M$ is smoothly foliated by immersed complex manifolds of dimension $n-1$. We shall denote by $\mathcal{F}_{M}$ such a Levi foliation.

If $M$ is moreover real analytic, then its local structure is very well understood: according to E. Cartan (see for instance [1, Section 1.7]), around each $p \in M$ we

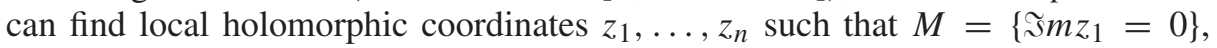
and consequently the leaves of $\mathcal{F}_{M}$ are $\left\{z_{1}=c\right\}, c \in \mathbb{R}$. In particular, the Levi foliation $\mathcal{F}_{M}$ extends on a neighbourhood of $p$ to a codimension one holomorphic foliation, with leaves $\left\{z_{1}=c\right\}, c \in \mathbb{C}$. It is then easy to see that these local extensions glue together, giving a codimension one holomorphic foliation $\mathcal{F}$ on some neighbourhood of $M$.

In this paper we are interested in finding a similar structure in the case of singular Levi-flat hypersurfaces, a subject sistematically initiated by Burns and Gong [2]. Let us firstly recall some terminology.

A closed subset $M \subset X$ is real analytic if it is locally defined by the vanishing of a (finite) collection of real analytic functions. A real analytic subset $M$ is irreducible if it cannot be expressed as $M=M_{1} \cup M_{2}$, with both $M_{1}$ and $M_{2}$ real 
analytic and different from $M$. Any real analytic subset can be decomposed (on relatively compact open subsets) into a finite collection of irreducible components. An irreducible real analytic subset $M$ has a well defined $\operatorname{dimension} \operatorname{dim}_{\mathbb{R}} M$, and it can be decomposed as a disjoint union $M=M_{\text {reg }} \cup M_{\text {sing, }}$, where: (i) $M_{\text {reg }}$ is nonempty and open in $M$, and it is formed by those points of $M$ around which $M$ is a smooth real analytic submanifold of $X$ of dimension $\operatorname{dim}_{\mathbb{R}} M$; (ii) $M_{\text {sing }}$ is a real analytic subset, all of whose irreducible components have dimension strictly smaller than $\operatorname{dim}_{\mathbb{R}} M$. Remark, however, that $M_{\text {reg }}$ may fail to be dense in $M$, as in the well known Whitney umbrella.

When $\operatorname{dim}_{\mathbb{R}} M=\operatorname{dim}_{\mathbb{R}} X-1=2 n-1$, or more generally each irreducible component of $M$ has dimension $2 n-1$, we shall call $M$ a real analytic hypersurface. In that case, we shall say that $M$ is Levi-flat if $M_{\text {reg }}$ is Levi-flat in the usual sense (Frobenius' integrability).

If $M \subset X$ is a Levi-flat real analytic hypersurface then we have on $M_{\text {reg }}$ the Levi foliation $\mathcal{F}_{M_{\text {reg }}}$. A natural question is: does this foliation extend holomorphically on some neighbourhood of the closure $\overline{M_{\text {reg }}}$ ? Of course, we need here to work, at least, with singular codimension one holomorphic foliations; see for instance [5] for the basic dictionary. Let us see some examples.

Example 1.1. Take a nonconstant holomorphic function $f: X \rightarrow \mathbb{C}$ and set $M=$ $\{\Im m f=0\}$. Then $M$ is Levi-flat and $M_{\text {sing }}$ is the set of critical points of $f$ lying on $M$. Leaves of the Levi foliation on $M_{\text {reg }}$ are given by $\{f=c\}, c \in \mathbb{R}$. Of course, this Levi foliation can be extended to a singular holomorphic foliation on the full $X$, generated by the kernel of $d f$. More complicated examples can be obtained by taking $M=\{F \circ f=0\}$ for some real analytic function $F: \mathbb{C} \rightarrow \mathbb{R}$, or by starting with a meromorphic $f[2]$.

Example 1.2. In $X=\mathbb{C}^{2}$ with coordinates $z=x+i y, w=s+i t$, consider the irreducible real analytic hypersurface $M$ defined by

$$
M=\left\{t^{2}=4\left(y^{2}+s\right) y^{2}\right\} .
$$

We have $M_{\text {sing }}=\{t=y=0\}$, a totally real plane in $\mathbb{C}^{2}$. Note that $\overline{M_{\text {reg }}} \cap M_{\text {sing }}=$ $\{t=y=0, s \geq 0\}$, a proper subset of $M_{\text {sing }}$. This hypersurface is Levi-flat: on $M_{\text {reg }}$, the leaves of $\mathcal{F}_{M_{\text {reg }}}$ are the complex curves

$$
L_{c}=\left\{w=(z+c)^{2}, \Im m z \neq 0\right\}, \quad c \in \mathbb{R} .
$$

For every $c \in \mathbb{R}$ the closure $\overline{L_{c}}=\left\{w=(z+c)^{2}\right\}$ cuts $M_{\text {sing }}$ along the real curve $\left\{s=(x+c)^{2}\right\}$. Thus, each point of $M_{\text {sing }} \cap\{s>0\}$ belongs to two closures $\overline{L_{c_{1}}}$ and $\overline{L_{c_{2}}}$, whereas each point of $M_{\text {sing }} \cap\{s<0\}$ belongs to no one. The foliation $\mathcal{F}_{M_{\text {reg }}}$ extends holomorphically to a neighbourhood of $M_{\text {reg: just choose a square }}$ root of $w$ on $\mathbb{C}^{2} \backslash\{t=0, s \geq 0\}$ (which is a neighbourhood of $M_{\text {reg }}$ ), and then take the holomorphic foliation generated there by $d w=2 \sqrt{w} d z$. However, it is clear that $\mathcal{F}_{M_{\text {reg }}}$ cannot be extended to neighbourhoods of points of $\overline{M_{\text {reg }}} \cap M_{\text {sing }}$, due to some "ramification" along $M_{\text {sing }}$ (or, more precisely, along the real line $\{y=t=$ 
$s=0\} \subset M_{\text {sing }}$ : the other points are not seriously problematic, for around them $M$ is the union of two smooth Levi-flat hypersurfaces intersecting transversely). In spite of this, we could say that $\mathcal{F}_{M_{\text {reg }}}$ can be extended in some weak or multiform sense, as solution of the implicit differential equation $\left(\frac{d w}{d z}\right)^{2}=4 w$.

Our main result is an extrapolation of this last example. The philosophy which is behind is that, even if sometimes it is impossible to extend $\mathcal{F}_{M_{\text {reg }}}$, it is always possible to extend it in a "microlocal" sense, i.e. after lifting to the cotangent bundle. In other words, $\mathcal{F}_{M_{\text {reg }}}$ can always be extended as a (transcendental) implicit differential equation, or web. This will be better explained in Section 2 below. For the moment, we state our result in the form of a "resolution theorem" for singular Levi-flat hypersurfaces.

Theorem 1.3. Let $X$ be a complex manifold of dimension $n$, and let $M \subset X$ be a Levi-flat real analytic hypersurface. Then there exist a complex manifold $Y$ of dimension n, a Levi-flat real analytic hypersurface $N \subset Y$, a (singular) codimension one holomorphic foliation $\mathcal{F}$ on $Y$ extending $\mathcal{F}_{N_{\text {reg }}}$, and a holomorphic map $\pi: Y \rightarrow X$, such that for some open $N_{0} \subset N$ :

(i) $\left.\pi\right|_{N_{0}}: N_{0} \rightarrow M_{\text {reg }}$ is an isomorphism;

(ii) $\left.\pi\right|_{\overline{N_{0}}}: \overline{N_{0}} \rightarrow \overline{M_{\text {reg }}}$ is a proper map.

In the Example 1.2 above, we may take $Y=\mathbb{C}^{2}, N$ a real hyperplane, and $\pi$ a quadratic map which sends complex lines in $N$ to the curves $\overline{L_{c}}, c \in \mathbb{R}$. Remark that here $N_{0}$ is not equal to $N_{\text {reg }}$, it is only a (open and dense) part of it. Unfortunately,

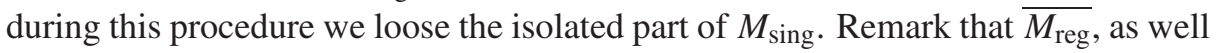
as $\overline{N_{0}}$, is not a real analytic subset, it is only subanalytic. It could be interesting to generalise the above theorem, in some way, to the subanalytic setting, but our method is strictly inside the real analytic world.

The theorem above can be seen as a first step toward a resolution procedure for singularities of Levi-flat hypersurfaces: the map $\pi$ is a sort of blow-up of $\overline{M_{\text {reg }}} \backslash$

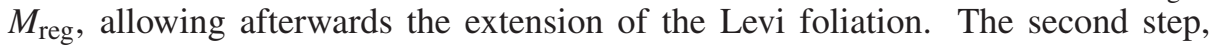
which for the moment requires $n \leq 3$, consists in applying the Desingularisation Theorem of Seidenberg $(n=2)$ or Cano $(n=3)[3,5]$ to the foliation $\mathcal{F}$. The third, and easy, step is the analysis of Levi-flat real analytic hypersurfaces which are tangent to a simple singularity of codimension one foliation. We leave the details to the interested reader.

The proof of Theorem 1.3 is based on quite elementary considerations concerning the complexification(s) of real analytic subsets (not only hypersurfaces) in complex manifolds, which in some sense stem from the seminal work of Diederich and Fornaess [6] and which are also exploited in [2].

\section{Lifting to the cotangent bundle}

In this section we give a more precise statement of our main result, and we reduce its proof to a general statement on the intrinsic complexification of certain real analytic 
subsets.

Consider, as before, a Levi-flat real analytic hypersurface $M$ in a complex manifold $X, \operatorname{dim}_{\mathbb{C}} X=n$. Let $P T^{*} X$ be the projectivised cotangent bundle of $X$ : a $\mathbb{C} P^{n-1}$-bundle over $X$, whose fibre $P T_{x}^{*} X$ over $x \in X$ will be thought as the set of complex hyperplanes in $T_{X} X$. Denote by $\pi$ the projection $P T^{*} X \rightarrow X$.

The regular part $M_{\text {reg }}$ of $M$ can be lifted to $P T^{*} X$ : just take, for every $x \in$ $M_{\text {reg }}$, the complex hyperplane

$$
T_{x}^{\mathbb{C}} M_{\text {reg }}=T_{x} M_{\text {reg }} \cap J\left(T_{x} M_{\text {reg }}\right) \subset T_{x} X .
$$

Call

$$
M_{\text {reg }}^{\prime} \subset P T^{*} X
$$

this lifting of $M_{\text {reg. }}$ Remark that it is no more a hypersurface: its (real) dimension $2 n-1$ is half of the real dimension of $P T^{*} X$. However, it is still "Levi-flat", in a sense which will be more precisely specified below.

Take now a point $y$ in the closure $\overline{M_{\text {reg }}^{\prime}}$, projecting on $X$ to a point $x \in \overline{M_{\text {reg }}}$.

Lemma 2.1. There exists, in a neighbourhood $U_{y} \subset P T^{*} X$ of $y$, a real analytic subset $N_{y}$ of dimension $2 n-1$ containing $M_{\mathrm{reg}}^{\prime} \cap U_{y}$.

(The notation suggests that $U_{y}$ and $N_{y}$ should be more properly considered as germs at $y$. Similarly for the proposition below.)

Proof. We choose local coordinates $z_{1}, \ldots, z_{n}, w_{1}, \ldots, w_{n-1}$ around $y$ such that $z_{1}, \ldots, z_{n}$ are coordinates around $x$ and $w_{1}, \ldots, w_{n-1}$ corresponds to the hyperplane $d z_{n}+\sum_{j=1}^{n-1} w_{j} d z_{j}=0$. Take a real analytic equation $f$ of $M$ around $x$, with $d f \neq 0$ on $M_{\text {reg. }}$. Then the real analytic subset defined around $y$ by the equations

$$
f=0 \quad, \quad w_{j}=\frac{\partial f}{\partial z_{j}} / \frac{\partial f}{\partial z_{n}} \quad, \quad j=1, \ldots, n-1
$$

contains $M_{\text {reg }}^{\prime}$ as an open subset. Indeed, $T^{\mathbb{C}} M_{\text {reg }}$ is the Kernel of $\partial f$. This analytic subset may have dimension larger than $2 n-1$ (for instance, it contains the full fibres over $M_{\text {sing }}$ ), but using a stratification of it we see that it contains a real analytic subset $N_{y}$ (a stratum) of dimension $2 n-1$ and which still contains $M_{\text {reg }}^{\prime}$.

We choose such a $N_{y}$ as the minimal one (as a germ at $y$ ), by taking intersections. We have $M_{\text {reg }}^{\prime} \cap U_{y} \subset\left(N_{y}\right)_{\text {reg }}$, but the inclusion may be strict, as in the Example 1.2 of the Introduction. Also, $N_{y}$ may have several irreducible components, but each one contains a part of $M_{\text {reg }}^{\prime} \cap U_{y}$, by minimality.

The crucial fact is now the following.

Proposition 2.2. There exists, in a neighbourhood $V_{y} \subset U_{y}$ of $y$, a complex analytic subset $Y_{y}$ of (complex) dimension $n$ containing $N_{y} \cap V_{y}$.

Let us deduce Theorem 1.3 from this proposition. 
First of all, $N_{y} \cap V_{y}$ is a real analytic hypersurface in $Y_{y}$, and it is Levi-flat because each irreducible component contains a Levi-flat piece [2, Lemma 2.2]. On $Y_{y}$, we have a natural codimension one holomorphic (singular) distribution of hyperplanes, given by the restriction of the canonical contact structure of $P T^{*} X$. At points of $M_{\text {reg }}^{\prime}$, this codimension one distribution coincides (by tautology of the contact form) with $T^{\mathbb{C}} M_{\text {reg. }}$. In other words and more precisely, around points of $M_{\text {reg }}^{\prime} \cap V_{y}$ the projection $\pi: Y_{y} \rightarrow X$ is a biholomorphism, sending $M_{\text {reg }}^{\prime}$ to $M_{\text {reg }}$, thus $Y_{y}$ appears as the graph of a local holomorphic section of $P T^{*} X$ extending the real analytic section $M_{\text {reg }} \ni x^{\prime} \mapsto y^{\prime}=T_{x^{\prime}}^{\mathbb{C}} M_{\text {reg }} \in M_{\text {reg }}^{\prime}$. This local holomorphic section is a local hyperplane distribution on $X$. When lifted to $Y_{y}$ by the same $\pi$, this distribution becomes a distribution on $Y_{y}$ coinciding with the restriction of the contact structure.

Now, this distribution is integrable, because it is integrable on a real hypersurface, hence it defines a codimension one (singular) foliation $\mathcal{F}_{y}$ on $Y_{y}$. Clearly, this foliation $\mathcal{F}_{y}$ extends the Levi foliation of $\left(N_{y}\right)_{\text {reg }} \cap V_{y}$, because so is for $M_{\text {reg }}^{\prime} \cap V_{y}$ and each irreducible component of $N_{y} \cap V_{y}$ contains a portion of $M_{\text {reg }}^{\prime} \cap V_{y}$.

These local constructions are sufficiently canonical to be patched together, when $y$ varies on $\overline{M_{\text {reg }}^{\prime}}$ : if $Y_{y_{1}} \subset V_{y_{1}}$ and $Y_{y_{2}} \subset V_{y_{2}}$ are as above, with $M_{\text {reg }}^{\prime} \cap$ $\left(V_{y_{1}} \cap V_{y_{2}}\right) \neq \emptyset$, then $Y_{y_{1}} \cap\left(V_{y_{1}} \cap V_{y_{2}}\right)$ and $Y_{y_{2}} \cap\left(V_{y_{1}} \cap V_{y_{2}}\right)$ have some common irreducible components containing $M_{\text {reg }}^{\prime} \cap\left(V_{y_{1}} \cap V_{y_{2}}\right)$, and so $Y_{y_{1}}$ and $Y_{y_{2}}$ can be glued by identifying those components. In this way, we obtain an analytic space $Y$ of dimension $n$, with a Levi-flat hypersurface $N$ and a holomorphic foliation $\mathcal{F}$, enjoying all the properties stated in Theorem 1.3; the map $\pi: Y \rightarrow X$ is deduced from the projection of $P T^{*} X$ to $X$. Finally, to get a smooth $Y$ we just replace the possibly singular $Y$ with a resolution $\widetilde{Y}$ of it, over which $N$ and $\mathcal{F}$ can be lifted. Note that on $Y$ the foliation $\mathcal{F}$ is locally defined by a holomorphic 1-form, the restriction to $Y$ of the contact form on $P T^{*} X$; therefore on $\widetilde{Y}$ the lifted foliation will be also locally defined by a holomorphic 1 -form, obtained by pulling-back the contact form under the resolution map.

Let us reformulate this proof from a slightly different point of view.

On some neighbourhood $X_{0} \subset X$ of $M_{\text {reg }}$ we have a holomorphic foliation $\mathcal{F}_{0}$ extending the Levi foliation $\mathcal{F}_{M_{\text {reg }}}$. The graph of this foliation is a complex manifold $Y_{0} \subset P T^{*} X$ which projects bihlomorphically to $X_{0}$ by $\pi$. This $Y_{0}$ contains $M_{\mathrm{reg}}^{\prime}$, as a Levi-flat hypersurface. The main point is then to extend $Y_{0}$ to a neighbourhood of $\overline{M_{\text {reg }}^{\prime}}$, and this is the content of Proposition 2.2. In this way, an extension problem for foliations (or, more appropriately, for webs) has been transformed into an extension problem for complex analytic subspaces.

Remark 2.3. The analytic subset $Y_{y}$ can be seen as an implicit differential equation on $X$, around $x$. But there are two quite different situations. If $\pi: Y_{y} \rightarrow X$ is a finite map, then (by Weierstrass) $Y_{y}$ has an equation which is polynomial in the vertical variables $w_{1}, \ldots, w_{n-1}$, so that the associated implicit differential equation is "algebraic in the derivatives". In some sense, even if there is a ramification we could say that this situation is "not-too-singular", from the differential equation point of view. If on the contrary $\pi: Y_{y} \rightarrow X$ is not finite over $x$, we don't 
know if such an algebraicity in the derivatives persists, i.e. if $Y_{y}$ can be analytically continued to a neighbourhood of the full fibre over $x$.

Example 2.4. Consider the subset $M \subset \mathbb{C}^{2}$ defined by

$$
M=\bigcup_{\lambda \in \mathbb{S}^{1}}\left\{z_{2}=\lambda z_{1}+\varphi(\lambda) z_{1}^{2}\right\}
$$

where $\varphi: \mathbb{S}^{1} \rightarrow \mathbb{C}$ is a real analytic function. Outside the origin (and close to it) $M$ is real analytic, smooth, and Levi-flat. Thus $M \backslash\{0\}$ can be lifted to $P T^{*} \mathbb{C}^{2}=$ $\mathbb{C}^{2} \times \mathbb{C} P^{1}$, and the closure of this lifting is a smooth real analytic threefold $N$ which cuts the fibre over 0 along a smooth circle. The cooking recipe to obtain the complex surface $Y$ containing $N$ is the following. We differentiate the complex curves contained in $M$,

$$
d z_{2}=\left(\lambda+2 \varphi(\lambda) z_{1}\right) d z_{1},
$$

and then we "eliminate" $\lambda$ from the two equations $z_{2}=\lambda z_{1}+\varphi(\lambda) z_{1}^{2}$ and $\frac{d z_{2}}{d z_{1}}=$ $\lambda+2 \varphi(\lambda) z_{1}$, thus obtaining an analytic relation between $z_{1}, z_{2}$ and $w_{1}=-\frac{d z_{2}}{d z_{1}}$. It is quite clear that such an equation will be algebraic (in $w_{1}$ ) if and only if $\varphi$ is algebraic, i.e. $\varphi(\lambda)=\sum_{j=-\ell}^{\ell} a_{j} \lambda^{j}$. However, we believe that this algebraicity condition on $\varphi$ should be also necessary and sufficient for the real analyticity of $M$ at the origin. Thus, if $M$ is real analytic at 0 then we would obtain an implicit differential equation which is algebraic in the derivatives, and the question of the previous Remark remains unanswered. Note, however, that whatever $\varphi$ is, the subset $N$ is everywhere analytic; hence our method, which is based on the analyticity of $N$ and not of $M$, is unfortunately rather useless for these type of subtle problems.

Proposition 2.2 is a particular case of a more general fact, for which it is convenient to introduce some more terminology.

Let $Z$ be a complex manifold of dimension $m$. Let $N \subset Z$ be a real analytic subset of dimension $2 n-1$. We shall say that $N$ is a Levi-flat real analytic subset if its regular part is foliated by complex submanifolds of (complex) dimension $n-1$. As in the hypersurface case, we shall denote by $\mathcal{F}_{N_{\text {reg }}}$ this Levi foliation on $N_{\text {reg }}$; it is real analytic and of real codimension one (in $N_{\text {reg }}$ ). This definition can be reformulated in the language of CR geometry [1, Chapter I]: $N_{\text {reg }}$ is a CR submanifold of CR dimension $n-1$ (or CR codimension one) whose complex tangent bundle $T^{\mathbb{C}} N_{\text {reg }}=T N_{\text {reg }} \cap J\left(T N_{\text {reg }}\right)$ is integrable. It is easy to see, as in the hypersurface case [2, Lemma 2.2], that it is sufficient to check the integrability only on some open nonempty subset $N_{0} \subset N_{\text {reg. }}$.

As in the hypersurface case, the local structure of $N_{\text {reg }}$ is well understood [1, Section 1.8]: around every $z \in N_{\text {reg }}$ there are local holomorphic coordinates $z_{1}, \ldots, z_{m}$ on $Z$ such that

$$
N_{\text {reg }}=\left\{\Im m z_{1}=0, z_{m-n}=\ldots=z_{m}=0\right\} .
$$

In particular, around $z$ there is a canonically defined complex submanifold of $Z$, of dimension $n$, which contains $N_{\text {reg }}$ :

$$
Y_{0}=\left\{z_{m-n}=\ldots=z_{m}=0\right\} .
$$


This $Y_{0}$ is called intrinsic complexification of $N_{\text {reg }}$ (around $z$ ). It is the smallest complex submanifold containing $N_{\text {reg. }}$. Remark that $N_{\text {reg }}$ is a (smooth) Levi-flat hypersurface in $Y_{0}$, and the Levi foliation $\mathcal{F}_{N_{\text {reg }}}$ can be canonically extended to a holomorphic codimension one foliation $\mathcal{F}_{Y_{0}}$ on $Y_{0}$. This is the situation that we have already met in the proof of Theorem 1.3.

Now the basic result, from which Proposition 2.2 follows, states that this intrinsic complexification can be analytically prolonged around points of the closure $\overline{N_{\text {reg }}}$ :

Theorem 2.5. Let $Z$ be a complex manifold of dimension $m$, and let $N \subset Z$ be a Levi-flat real analytic subset of dimension $2 n-1$. Then, for every $z \in \overline{N_{\text {reg }}}$, there exists a neighbourhood $V \subset Z$ of $z$ and a complex analytic subset $Y \subset V$ of dimension $n$ which contains $N_{\text {reg }} \cap V$

The proof will be given in the next section, and it is based on the following idea. There exists a second type of complexification of $N$, which could be called extrinsic complexification [4]. It lives in a larger space, not inside $Z$, but it has the advantage that it can be defined around every point of $N$, not only $N_{\text {reg. }}$ The intrinsic complexification appears as a "projection" of the extrinsic one, and from the analytic extendability to singular points of the latter we shall deduce the analytic extendability of the former. In a vague sense, this is related to [6, Appendix].

It is however important to realize that this result holds because the CR codimension of $N_{\text {reg }}$ is one, and no more. Here is an example showing that an analogous statement for the higher CR codimensional case may fail. This is related to the fact that, whereas a holomorphic map from a complex curve is always "locally proper", the same is no more true for maps from higher dimensional complex manifolds, due to the phenomenon of "contraction of divisors".

Example 2.6. We work in $\mathbb{C}^{3}$, with coordinates $z_{1}, z_{2}, z_{3}$. Let $S \subset \mathbb{C}^{3}$ be the real analytic surface defined by

$$
\begin{gathered}
z_{2}=\left(\Re e z_{1}\right) z_{1} \\
z_{3}=e^{\Re e z_{1}} z_{1}
\end{gathered}
$$

Note that $S$ is smooth, being the graph over the $z_{1}$-axis of a smooth function $F$ : $\mathbb{C}_{z_{1}} \rightarrow \mathbb{C}_{z_{2}, z_{3}}$. At the point $0 \in S$ we have $T_{0}^{\mathbb{C}} S=T_{0} S$, a complex line, whereas at any other point $p \in S, p \neq 0$, we have $T_{p}^{\mathbb{C}} S=\{0\}$. Indeed, $0 \in \mathbb{C}_{z_{1}}$ is the only point where the differential of $F$ is $\mathbb{C}$-linear. Thus, $S_{0}=S \backslash\{0\}$ is a CR submanifold of CR dimension 0 and CR codimension 2. The "Levi foliation" is here the foliation by points.

Along $S_{0}$ we have the intrinsic complexification $Y_{0} \supset S_{0}$, which is a complex surface. However, this complex surface cannot be extended around the point 0 . To see this, observe that over $\left\{z_{1} \neq 0\right\}$ the surface $Y_{0}$ has equation

$$
z_{3}=e^{z_{2} / z_{1}} z_{1}
$$

which has an essential singularity at $z_{1}=0$. More geometrically, if we blow up $\mathbb{C}^{3}$ at the origin, $\widetilde{\mathbb{C}^{3}} \stackrel{b}{\rightarrow} \mathbb{C}^{3}$, then the closure of $b^{-1}\left(Y_{0}\right)$ has a trace on the exceptional 
divisor $b^{-1}(0) \simeq \mathbb{C} P^{2}$ which contains the complex curve with equation $w_{3}=e^{w_{2}}$, in affine coordinates $w_{2}=z_{2} / z_{1}, w_{3}=z_{3} / z_{1}$. The transcendency of this curve implies that $Y_{0}$ cannot be holomorphically extended at 0 .

\section{Complexification of Levi-flat subsets}

We start the proof of Theorem 2.5 by recalling some facts about (extrinsic) complexification of real analytic subsets [4], [1, Chapter X].

Let $Z$ be a complex manifold of dimension $m$. We shall denote by $Z^{*}$ the complex manifold conjugate to $Z$, that is obtained by replacing the complex structure $J$ of $Z$ with the opposite complex structure $-J$. If $A \subset Z$ is a complex analytic subset, then the same subset is also a complex analytic subset of $Z^{*}$, but with the opposite complex structure; it will be denoted by $A^{*}$. The diagonal $\Delta \subset Z \times Z^{*}$ is a totally real submanifold. The projections of $Z \times Z^{*}$ onto $Z$ and $Z^{*}$ will be respectively denoted by $\Pi$ and $\Pi^{*}$, and the antiholomorphic involution $Z \times Z^{*} \rightarrow Z \times Z^{*}$, $\left(z_{1}, z_{2}\right) \mapsto\left(z_{2}, z_{1}\right)$, will be denoted by $J$.

Let $N \subset Z$ be a real analytic subset of dimension $k$, and fix a point $z_{0} \in \overline{N_{\text {reg }}}$. Without loss of generality for our purposes, we will suppose that the germ of $N$ at $z_{0}$ is irreducible. Set

$$
N^{\Delta}=\left\{(z, z) \in Z \times Z^{*} \mid z \in N\right\} .
$$

Then in some sufficiently small neighbourhood of $\left(z_{0}, z_{0}\right)$, which we may assume equal to $Z \times Z^{*}$ by restricting $Z$, there exists an irreducible complex analytic subset $N^{\mathbb{C}}$ of dimension $k$ such that

$$
N^{\mathbb{C}} \cap \Delta=N^{\Delta}
$$

This $N^{\mathbb{C}}$ is called complexification of $N$ at $z_{0}$. It is obtained by complexifying the real analytic equations defining $N$, and it can be characterized as the smallest (germ at $\left(z_{0}, z_{0}\right)$ of) complex analytic subset containing $N^{\Delta}$. It enjoys the following fundamental symmetry property: $\mathrm{J}$ leaves $N^{\mathbb{C}}$ invariant, and $N^{\Delta}$ is the fixed point set of $\left.j\right|_{N} \mathbb{C}$.

A word of caution. A real analytic subset is not necessarily coherent [4]. This means that if $z_{1} \in \overline{N_{\text {reg }}}$ is another point, close to $z_{0}$, then we can still define the complexification $\widetilde{N^{\mathbb{C}}}$ of $N$ at $z_{1}$ (if the germ of $N$ at $z_{1}$ has several irreducible components, we complexify each one), however it may happen that $\widetilde{N^{\mathbb{C}}}$, defined in some neighbourhood of $\left(z_{1}, z_{1}\right)$, is smaller than the restriction of $N^{\mathbb{C}}$ to that neighbourhood. More precisely, it may happen that the germ of $\widetilde{N^{\mathbb{C}}}$ at $\left(z_{1}, z_{1}\right)$ is not the full germ of $N^{\mathbb{C}}$ at $\left(z_{1}, z_{1}\right)$, but only a union of certain irreducible components of it. The situation is even worse if we take $z_{1} \in N \backslash \overline{N_{\text {reg }}}$ : the local dimension of $N$ at $z_{1}$ is smaller than $k$, and thus the complexification of $N$ at $z_{1}$ has also smaller dimension; the germ of $\widetilde{N^{\mathbb{C}}}$ at $\left(z_{1}, z_{1}\right)$ is then a proper subset of the germ of $N^{\mathbb{C}}$. 
Suppose now that $N$ is Levi-flat, $k=2 n-1$. Take a regular point $z \in N_{\text {reg. }}$. As we saw in the previous section, in some neighbourhood $V_{z} \subset Z$ of $z$ there exists a unique smooth complex submanifold $Y_{z}$ of dimension $n$, which contains $N_{\text {reg }} \cap V_{z}$ and over which the Levi foliation extends as a holomorphic codimension one foliation $\mathcal{F}_{z}$. For a good choice of $V_{z}$, we may assume that the space of leaves $Y_{z} / \mathcal{F}_{z}$ is a disc $\mathbb{D}$, in which the Levi leaves correspond to points of $\mathbb{I}=(-1,1) \subset$ $\mathbb{D}$. Hence, we have a well defined Schwarz reflection

$$
s_{z}: Y_{z} / \mathcal{F}_{z} \rightarrow Y_{z} / \mathcal{F}_{z}
$$

with respect to $\mathbb{I}$.

Provided that $V_{z}$ is sufficiently small, the complexification of $N \cap V_{z}$ at $z$ is a smooth complex submanifold $N_{z}^{\mathbb{C}}$ of $U_{z}=V_{z} \times V_{z}^{*} \subset Z \times Z^{*}$, of dimension $2 n-1$, which in fact coincides with an irreducible component of $N^{\mathbb{C}} \cap U_{z}$. The following Lemma gives the precise relation between $Y_{z}$ and $N_{z}^{\mathbb{C}}$.

Lemma 3.1. The projection $\Pi$ induces a smooth holomorphic fibration of $N_{z}^{\mathbb{C}}$ over $Y_{z}$, and the fibre of $\Pi: N_{z}^{\mathbb{C}} \rightarrow Y_{z}$ over $z^{\prime} \in Y_{z}$ is the Schwarz reflection of the leaf of $\mathcal{F}_{z}$ through $z^{\prime}$ (with the opposite complex structure).

Proof. In suitable local coordinates $z_{1}, \ldots, z_{m}$ we have

$$
\begin{aligned}
N_{\mathrm{reg}} & =\left\{\Im m z_{1}=0, z_{m-n}=\ldots=z_{m}=0\right\} \\
Y_{z} & =\left\{z_{m-n}=\ldots=z_{m}=0\right\} .
\end{aligned}
$$

If $z^{\prime} \in Y_{z}$, then the leaf through $z^{\prime}$ is

$$
L_{z^{\prime}}=\left\{z_{1}=a, z_{m-n}=\ldots=z_{m}=0\right\}
$$

where $a$ is the $z_{1}$-coordinate of $z^{\prime}$, and its Schwarz reflection is

$$
s_{z}\left(L_{z^{\prime}}\right)=\left\{z_{1}=\bar{a}, z_{m-n}=\ldots=z_{m}=0\right\} .
$$

Local coordinates on $Z \times Z^{*}$ are provided by $z_{1}, \ldots, z_{m}, \bar{z}_{1}, \ldots, \bar{z}_{m}$, and in these coordinates

$$
N_{z}^{\mathbb{C}}=\left\{z_{1}=\bar{z}_{1}, z_{m-n}=\ldots=z_{m}=\bar{z}_{m-n}=\ldots=\bar{z}_{m}=0\right\} .
$$

We therefore see that $N_{z}^{\mathbb{C}}$ projects by $\Pi$ to $Y_{z}$, and the fibre over $z^{\prime}$ is

$$
\left\{\bar{z}_{1}=a, \bar{z}_{m-n}=\ldots=\bar{z}_{m}=0\right\}=s_{z}\left(L_{z^{\prime}}\right)^{*} .
$$

Of course, a similar statement holds also for the second projection $\Pi^{*}$. Thus, $N_{z}^{\mathbb{C}}$ is a smooth complex hypersurface in $Y_{z} \times Y_{z}^{*} \subset Z \times Z^{*}$, with a double fibration over $Y_{z}$ and $Y_{z}^{*}$. 
Note that for every $z^{\prime} \in Y_{z}$ we can also consider the fibre over $z^{\prime}$ of the full $N^{\mathbb{C}}$, i.e. the intersection $N^{\mathbb{C}} \cap\left(\left\{z^{\prime}\right\} \times Z^{*}\right)$. This is an analytic subset of $Z^{*}$ which extends the leaf $s_{z}\left(L_{z^{\prime}}\right)^{*} \subset V_{z}^{*}$. In particular, each leaf of $\mathcal{F}_{z}$ has an analytic continuation to the full $Z$. This fundamental fact was discovered in [6], in a different context, and it was our starting point.

Let us now see what happens at the singular point $z_{0}$.

For every $p \in N^{\mathbb{C}}$ consider the vertical fibre through $p$

$$
F_{p}^{*}=\left\{q \in N^{\mathbb{C}} \mid \Pi(q)=\Pi(p)\right\}
$$

and define

$$
d(p)=\operatorname{dim}_{p}\left(F_{p}^{*}\right)
$$

where $\operatorname{dim}_{p}$ denotes the local dimension at $p$ (if $F_{p}^{*}$ is reducible at $p$, we take the maximal dimension among irreducible components). According to Cartan or Remmert [7, Section V.3], the function $d$ on $N^{\mathbb{C}}$ is Zariski semicontinuous, thus equal to some constant $d_{0}$ over some Zariski open and dense $\breve{N}^{\mathbb{C}} \subset N^{\mathbb{C}}$ and strictly greater than $d_{0}$ over $N^{\mathbb{C}} \backslash \breve{N}^{\mathbb{C}}$. By Lemma 3.1, we have $d_{0}=n-1$, and moreover $\breve{N}^{\mathbb{C}}$ contains generic points of $N_{\text {reg }}^{\Delta}$ (here we say "generic", and not "all", because at special points $(z, z) \in N_{\text {reg }}^{\Delta}$ the germ of $N^{\mathbb{C}}$ could have a second irreducible component different from $N_{z}^{\mathbb{C}}$ ).

Set $p_{0}=\left(z_{0}, z_{0}\right)$.

If $d\left(p_{0}\right)=d_{0}=n-1$, then by the Rank theorem of Remmert [7, Section V.6] there exists a neighbourhood $U_{z_{0}} \subset Z \times Z^{*}$ of $p_{0}$ such that $\Pi\left(N^{\mathbb{C}} \cap U_{z_{0}}\right)$ is a complex analytic subset $Y_{z_{0}}$ of $V_{z_{0}}=\Pi\left(U_{z_{0}}\right)$, of dimension $n$. The situation is not very different from the one of Lemma 3.1. And we are just in the conclusion of Theorem 2.5: obviously $Y_{z_{0}}$ contains the previously defined $Y_{z}$, for every $z \in$ $N_{\text {reg }} \cap V_{z_{0}}$.

Hence, we shall suppose from now on that $d\left(p_{0}\right)=l \geq n$. Thus the vertical fibre $F_{p_{0}}^{*}$ contains an irreducible component $Y^{*} \subset Z^{*}$ of dimension $l$, passing through $p_{0}$. Its conjugate $Y=J\left(Y^{*}\right) \subset Z$ is an irreducible component of the horizontal fibre $F_{p_{0}}=\left\{q \in N^{\mathbb{C}} \mid \Pi^{*}(q)=\Pi^{*}\left(p_{0}\right)\right\}$. In the following, we shall consider $Y$ sometimes as a (horizontal) subset of $N^{\mathbb{C}}$, sometimes as a subset of the base $Z$.

Obviously, $Y \subset Z$ is contained in $\Pi\left(N^{\mathbb{C}}\right)$. Because $\operatorname{dim} N^{\mathbb{C}}=2 n-1$ and $d_{0}=n-1$, the image of $N^{\mathbb{C}}$ by $\Pi$ is a countable union of local analytic subsets of dimension $\leq n$ (by iterated application of the Rank theorem). Thus $Y$ cannot have dimension larger than $n$, and so

$$
\operatorname{dim} Y=n
$$

To complete the proof, we will show that $Y$ contains $\Pi\left(N^{\mathbb{C}} \cap U_{z_{0}}\right)$, for some neighbourhood $U_{z_{0}}$ of $z_{0}$ (thus, after all, $Y$ is equal to that projection, and so $Y$ is in fact the unique irreducible component of dimension $n$ of the horizontal fibre). Because $Y$ is closed, it is sufficient to verify this inclusion for $\Pi\left(\breve{N}^{\mathbb{C}} \cap U_{z_{0}}\right)$. 
Note that $Y \subset N^{\mathbb{C}}$ is not contained in $N^{\mathbb{C}} \backslash \breve{N}^{\mathbb{C}}$, for dimensional reasons: because $Y$ is horizontal of dimension $n$ and each vertical fibre through $N^{\mathbb{C}} \backslash \breve{N}^{\mathbb{C}}$ has dimension $\geq n, N^{\mathbb{C}}$ would have dimension $\geq 2 n$. Thus, $Y$ intersects $\breve{N}^{\mathbb{C}}$. If $p=\left(z, z_{0}\right)$ is a point of $Y \cap \breve{N}^{\mathbb{C}}$, then (by the Rank theorem) the image by $\Pi$ of a small neighbourhood of $p$ in $N^{\mathbb{C}}$ is an analytic subset $Y_{z} \subset V_{z} \subset Z$ of dimension $n$. The germ of $Y_{z}$ at $z$ could have several irreducible components, but at least one of them is contained in $Y$, because $Y \cap V_{z} \subset Y_{z}$ and $\operatorname{dim} Y=\operatorname{dim} Y_{z}$. By a connectivity argument (recall that $N^{\mathbb{C}}$ is irreducible, and therefore $\breve{N}^{\mathbb{C}}$ is connected), we see that $Y$ contains all the points arising from the projection of $\breve{N}^{\mathbb{C}}$ to $Z$, as claimed. section.

Let us conclude by looking again at the (counter) example of the previous

Example 3.2. Take again the real analytic surface $S$ in $\mathbb{C}^{3}$ with equation

$$
\begin{aligned}
& z_{2}=\left(\Re e z_{1}\right) z_{1} \\
& z_{3}=e^{\Re e z_{1}} z_{1}
\end{aligned}
$$

which, outside 0 , is a CR submanifold of CR dimension 0 and $\mathrm{CR}$ codimension 2. Its complexification is the complex analytic surface $S^{\mathbb{C}}$ in $\mathbb{C}^{3} \times \mathbb{C}^{3 *}$ with equation (setting $\bar{z}_{j}=w_{j}$ )

$$
\begin{array}{ll}
z_{2}=\frac{z_{1}+w_{1}}{2} z_{1}, & w_{2}=\frac{z_{1}+w_{1}}{2} w_{1} \\
z_{3}=e^{\frac{z_{1}+w_{1}}{2}} z_{1}, & w_{3}=e^{\frac{z_{1}+w_{1}}{2}} w_{1} .
\end{array}
$$

The projection of $S^{\mathbb{C}}$ to the first factor $\mathbb{C}^{3}$ is not analytic: something like $z_{3}=$ $e^{z_{2} / z_{1}} z_{1}$, for $z_{1} \neq 0$. The horizontal fibre through $0 \in S^{\mathbb{C}}$ is the complex curve $C \subset \mathbb{C}^{3}$ given by

$$
z_{2}=\frac{1}{2} z_{1}^{2} \quad, \quad z_{3}=e^{\frac{1}{2} z_{1}} z_{1}
$$

It is, of course, contained in the projection of $S^{\mathbb{C}}$ but not equal to. The dimensional arguments used several times in the proof of Theorem 2.5 cannot be used here.

\section{References}

[1] M. S. Baouendi, P. Ebenfelt and L. P. Rothschild, "Real Submanifolds in Complex Space and their Mappings", Princeton Mathematical Series 47, 1999.

[2] D. BURns and X. Gong, Singular Levi-flat real analytic hypersurfaces, Amer. J. Math. 121 (1999), 23-53.

[3] F. CANO, Reduction of the singularities of codimension one singular foliations in dimension three, Ann. of Math. 160 (2004), 907-1011.

[4] H. CARTAn, Variétés analytiques réelles et variétés analytiques complexes, Bull. Soc. Math. France 85 (1957), 77-99. 
[5] D. Cerveau, Feuilletages holomorphes de codimension 1. Réduction des singularités en petites dimensions et applications, Panor. Synthèses 8 (1999), 11-47.

[6] K. Diederich and J. E. Fornaess, Pseudoconvex domains with real-analytic boundary, Ann. of Math. 107 (1978), 371-384.

[7] S. LoJAsiewicz, "Introduction to Complex Analytic Geometry", Birkhäuser, 1991.

IMB - CNRS UMR 5584

9 Avenue Savary

21078 Dijon, France 